https://doi.org/10.18359/ravi.3368

\title{
Sistemas de redes sociales docentes: estudio documental mediante la cartografía conceptual ${ }^{1}$
}

\author{
María Isabel Ramírez Ochoa ${ }^{2}$, Araceli de Jesús Cázares Durán ${ }^{3}$ y Jesús Eduardo Torres Ortiz ${ }^{4}$
}

Sometido: 14 marzo de 2018

Evaluado: 30 abril de 2018

Aceptado: 2 mayo de 2018
Referencia: Ramírez-Ochoa, M.I., Cázares-Durán, A. de J. y Torres-Ortiz, J.E. (2018). Sistemas de redes sociales docentes: estudio documental mediante la cartografía conceptual. Revista Academia y Virtualidad, 11(2),18-37.

\section{Resumen}

Se realizó una investigación documental sobre el estado de conocimientos de los sistemas virtuales para albergar redes sociales docentes, mediante los ejes de análisis de la cartografía conceptual. Se examinó el contenido de un total de cuarenta y un documentos publicados entre los años 2002 y 2017, a nivel nacional e internacional, con la finalidad de redefinir su concepción, presencia y atribuciones en los procesos de educación continua docentes. Los resultados muestran que estas plataformas posibilitan la gestión de comunidades educativas complejas, en las que se construyen colectivamente contenidos de capital cultural docente, y que la intervención docente en estos espacios, a través de un procedimiento congruente con su metodología emergente, contribuye a formar sociedades de conocimiento.

Palabras clave: medios sociales; redes sociales; conceptualización; formación continua docente.

\footnotetext{
${ }^{1}$ Artículo de investigación científica y tecnológica

${ }^{2}$ Docente, investigadora y coordinadora del programa de Maestría en Educación de la Escuela Normal Experimental de El Fuerte, extensión Mazatlán. Investigadora del Centro de Investigación e Innovación Educativa del Sistema Valladolid, en México. Doctora en Tecnologías Avanzadas para la Educación y Magíster en Educación Ambiental. Correo electrónico: isabel.ramirez@sistemavalladolid.com

${ }^{3}$ Estudiante de la Maestría en Educación de la Escuela Normal Experimental de El Fuerte, extensión Mazatlán, en México. Correo electrónico: aracely.cazares90@gmail.com ${ }^{4}$ Estudiante de la Maestría en Educación de la Escuela Normal Experimental de El Fuerte, extensión Mazatlán, en México. Correo electrónico:jesuseduardotorresortiz@gmail.com
} 


\title{
Instructional Social Network Systems: A Documentary Research Study through Conceptual Mapping ${ }^{1}$
}

\section{María Isabel Ramírez Ochoa ${ }^{2}$, Araceli de Jesús Cázares Durán ${ }^{3}$ and Jesús Eduardo Torres Ortiz ${ }^{4}$}

\section{Reference:}

Ramírez-Ochoa, M.I.; Cázares-Durán, A. de J. and Torres-Ortiz, J.D. (2018) "Instructional Social Network Systems: A Documentary Study through Conceptual Mapping”. Academia y Virtualidad Journal 11, (2), p-p

\begin{abstract}
:
This study describes a documentary research study by focusing on the current knowledge levels of virtual systems for hosting instructional social networks based on the different axes used in conceptual mapping analysis. Based on this context, the content from 41 documents published between 2002 and 2017 at both the national and international levels was examined to redefine its conception, presence, and competences in continuous teaching education processes. The results denote that these platforms facilitate the management of complex educational communities wherein content is collectively developed for ensuring cultural capital in teaching and that adequate intervention from educators within these spaces by following a procedure consistent with the emerging methodology contributes to the formation of knowledge societies.
\end{abstract}

\section{Keywords:}

Social Media, Social Networks, Conceptualization, Continuous Teacher Education

\footnotetext{
${ }^{1}$ A Scientific and Technological Research Paper

${ }^{2}$ Professor, Researcher, and Coordinator, Master's Degree Program in Education, Teacher's Experimental Training School of El Fuerte at Mazatlan. Researcher, Center for Research in Educational Innovation, Valladolid, Mexico. Ph.D. in Advanced Educational Technology. M.A.Ed in Environmental Education. E-mail: isabel. ramirez@sistemavalladolid.com

${ }^{3}$ Teacher's Experimental Training School of El Fuerte at Mazatlan. Currently pursuing a master's degree in Education. Mexico. E-mail: aracely.cazares90@gmail.com

${ }^{4}$ Teacher's Experimental Training School of El Fuerte at Mazatlan. Currently pursuing a master's degree in Education. Mexico. E-mail: esuseduardotorresortiz@gmail.com
} 


\section{Sistemas de Redes Sociais Docentes: Um Estudo Documental através da Cartografia Conceitual ${ }^{1}$}

María Isabel Ramírez Ochoa ${ }^{2}$, Araceli de Jesús Cázares Durán ${ }^{3}$ e Jesús Eduardo Torres Ortiz ${ }^{4}$

\section{Referência:}

Ramírez-Ochoa, M.I.; Cázares-Durán, A. de J. and Torres-Ortiz, J.D. (2018) "Sistemas de Redes Sociais Docentes: Um Estudo Documental através da Cartografia Conceitual". Academia y Virtualidad Journal 11, (2), p-p

\section{Resumo:}

Foi realizada uma investigação documental sobre o estado do conhecimento de sistemas virtuais para abrigar redes sociais docentes através dos eixos de análise da cartografia conceitual. Analisou-se o conteúdo de um total de 41 documentos publicados entre 2002 e 2017 em nível nacional e internacional, a fim de redefinir sua concepção, presença e atribuições nos processos docentes de ensino de educação continuada. Os resultados mostram que essas plataformas possibilitam o gerenciamento de comunidades educacionais complexas no qual os conteúdos do capital cultural docente são construídos coletivamente e que, a intervenção docente a esses espaços através de um procedimento consistente com sua metodologia emergente contribui para formar sociedades de conhecimento.

\section{Palavras chave:}

Mídia Social, Rede Social, Conceitualização, Educação Contínua Docente

\footnotetext{
${ }^{1}$ Artigo de pesquisa científica e tecnológica

${ }^{2}$ Professor, Pesquisador e Coordenador do Programa de mestrado em educação da Escola Normal Experimental de El Fuerte, extensão de Mazatlan. Pesquisador do Centro de Investigação e Inovação Educativa do Sistema Valladolid, México. Doutor em Tecnologias Avançadas para a Educação e Mestre em Educação Ambiental. E-mail: isabel.ramirez@sistemavalladolid.com

${ }^{3}$ Escola Normal Experimental de El Fuerte, extensão Mazatlán. Estudante de mestrado em educação. México. E-mail: aracely.cazares90@gmail.com

${ }^{4}$ Escola Normal Experimental de El Fuerte, extensão Mazatlán. Atualmente mestranda em Educação, México.

E-mail: esuseduardotorresortiz@gmail.com
} 


\section{Introducción}

Las particularidades bidireccionales en la Web 2.0 convierten el ambiente virtual en un escenario comunicativo e interactivo de construcción social, que posibilita la modelación y la renovación colectiva de contenidos digitales (textos, imágenes, videos y audios). En este contexto se transita por medio de un conjunto de herramientas o aplicaciones tecnológicas, que configuran una constelación en función de cuatro orientaciones pedagógicas: recuperar información, crear recursos, compartir recursos y redes sociales (Scopeo, 2009). Estas últimas, también denominadas sistemas virtuales de comunicación social, plataformas virtuales para albergar redes sociales o Sitios de Redes Sociales (SRS o, en inglés, Social Network Sites, SNS), han adquirido relevancia en la investigación educativa: por ejemplo, la hemeroteca virtual Red de Revistas Científicas de América Latina y el Caribe, España y Portugal (Redalyc) contiene más de 417.575 artículos en español sobre el tema, desde el año 2000. Existen, además, estudios que han analizado el uso de los sRs como un medio formativo en la educación superior, mediante aproximaciones de corte cuantitativo (internacionales) y cualitativo (nacionales) (Fernández, Rodríguez y Haya, 2017; Navarro, Cuevas y Martínez, 2017), así como investigaciones que se clasifican en cinco temáticas: diseño de plataformas virtuales para redes sociales educativas; análisis de los SRS como medios educativos; utilización discente en contextos educativos; uso institucional, y utilización docente (Fernández, Rodríguez y Haya, 2017).

Los profesores son propensos a utilizar los SRS generales (Twitter) para publicar contenidos críticos y de controversia sobre los derechos civiles (temas relacionados con género, orientación sexual, raza y violencia) y temáticas de historia, matemáticas y alfabetización (Veletsianos y Kimmons, 2013). También suelen utilizar Facebook como un medio para fomentar relaciones positivas con los estudiantes (García, Froment, Bohórquez y Vieira, 2017); en respuesta a estas, se ha expuesto que los estudiantes aprecian a los profesores que se presentan auténticos en los ambientes virtuales (Mazer, Murphy y Simonds, 2009). Igualmente se hace uso de los SRS para investigadores (Researchgate, Academia.edu), pues estas plataformas son elegidas por los profesores para recabar, difundir documentos académicos, buscar impacto y aumentar el número de citas de sus producciones. De ahí que la administración de estas plataformas se haya agregado al conjunto de actividades que habitualmente realiza el docente-investigador (Veletsianos y Kimmons, 2013), ya sea para su actualización, la promoción de su trabajo o la comunicación con otro colega (García, Froment, Bohórquez y Vieira, 2017).

Aunque se ha identificado que los sRs ofrecen la oportunidad de romper las fronteras entre el aprendizaje formal e informal, la apreciación de estos como herramientas de gestión de conocimiento es incipiente, dado que son calificados solo como artefactos tecnológicos, lo que limita su concepción en procesos de formación continua docente (Fernández, Rodríguez y Haya, 2017). Por lo que sería conveniente revisar los estudios realizados desde la perspectiva de análisis, que ofrece la metodología de la cartografía conceptual (Tobón, 2013), aproximación que vislumbra las posibilidades formativas de las plataformas virtuales para redes sociales docentes o Sistemas de Redes Sociales Docentes (SRSD).

El propósito de este estudio consistió en analizar y sistematizar el contenido de diferentes fuentes sobre el estado de conocimiento de los Sistemas de Redes Sociales Docentes, mediante los ejes de análisis de la cartografía conceptual, con la finalidad de redefinir su concepción y atribuciones, diferenciar esta red de otras SRS, identificar su metodología emergente e ilustrar un ejemplo de su aplicación para la formación continua docente. 


\section{Metodología}

Se desarrolló un estudio cualitativo basado en el análisis documental (Pinto y Gálvez, 1996), que permitió organizar la información recabada de fuentes primarias y secundarias, en torno al concepto Sistema de Red Social Docente; se analizó el contenido de esta (Bardin, 1996; Krippendorff, 1990; Mayring, 2000; Pérez, 1994), y se generó el contenido concerniente a cada una de las categorías de análisis (Pérez, 1994), de acuerdo con la cartografía conceptual (Tobón, 2004, 2012). Tal cartografía define el seguimiento de ocho ejes de análisis: noción, categorización, caracterización, diferenciación, división, vinculación, metodología y ejemplificación, así como preguntas orientadoras emergentes de aquellos (ver tabla 1).

\section{Fases del estudio}

La investigación se llevó a cabo siguiendo las siguientes fases de aplicación de la cartografía conceptual, lo cual permitió enriquecer la información rescatada:

1. Se buscó información en fuentes primarias y secundarias que abordaran el concepto "Sistema de Red Social Docente", apoyándose en Google Académico y Redalyc, así como en artículos y revistas.

Tabla 1.

Ejes de la cartografía conceptual del término Sistema de Red Social Docente.

\begin{tabular}{|c|c|c|}
\hline Eje & Preguntas orientadoras & Componentes \\
\hline Noción & $\begin{array}{l}\text { ¿Cuál es la definición de Sistema de } \\
\text { Red Social Docente? }\end{array}$ & $\begin{array}{l}\text { - Etimología del término. } \\
\text { - Desarrollo histórico del SRSD. } \\
\text { - Definición actual. }\end{array}$ \\
\hline Categorización & $\begin{array}{l}\text { ¿Cuál es la categoría superior a la que } \\
\text { pertenecen los Sistemas de Redes } \\
\text { Sociales Docentes? }\end{array}$ & $\begin{array}{l}\text { - Ubicación del SRSD en las ciencias de la } \\
\text { educación } \\
\text { - Las teorías educativas asociadas a este } \\
\text { concepto. }\end{array}$ \\
\hline Caracterización & $\begin{array}{l}\text { ¿Cuáles son las características de } \\
\text { los Sistemas de Redes Sociales } \\
\text { Docentes? }\end{array}$ & $\begin{array}{l}\text { - Características comunicativas de los } \\
\text { SRSD. }\end{array}$ \\
\hline Diferenciación & \begin{tabular}{|l} 
¿Cuáles son las particularidades \\
distintivas de los Sistemas de Red \\
Social Docente?
\end{tabular} & $\begin{array}{l}\text { - Aspecto distintivo de los SRSD frente a } \\
\text { otros SRS. }\end{array}$ \\
\hline Clasificación & $\begin{array}{l}\text { ¿Cuáles son los tipos de Sistemas de } \\
\text { Redes Sociales Docentes? }\end{array}$ & $\begin{array}{l}\text { - Tipos y rasgos particulares de los } \\
\text { diferentes SRSD. }\end{array}$ \\
\hline Vinculación & $\begin{array}{l}\text { ¿Cómo se vinculan los Sistemas de } \\
\text { Redes Sociales Docentes con otros } \\
\text { enfoques o perspectivas por fuera } \\
\text { de la categorización? }\end{array}$ & $\begin{array}{l}\text { - Relación de los SRSD con otros conceptos } \\
\text { emergentes de la Web } 2.0 \text {. }\end{array}$ \\
\hline Metodología & $\begin{array}{l}\text { ¿Cómo se aplican los Sistemas de } \\
\text { Redes Sociales Docentes? }\end{array}$ & $\begin{array}{l}\text { - Pasos o elementos generales para abordar } \\
\text { problemas, contextualizados en el empleo } \\
\text { de los SRSD. }\end{array}$ \\
\hline Ejemplificación & $\begin{array}{l}\text { ¿Cuál puede ser un ejemplo } \\
\text { significativo del uso de un Sistema } \\
\text { de Red Social Docente? }\end{array}$ & $\begin{array}{l}\text { - Ejemplo concreto que ilustre la } \\
\text { aplicación del concepto y aborde los pasos } \\
\text { de la metodología construida para los SRSD. }\end{array}$ \\
\hline
\end{tabular}

Fuente: adaptado de Tobón et ál. (2015). 
2. Se seleccionó y se hizo un análisis de la información encontrada en las diferentes fuentes y documentos que cumplieran con la conceptualización buscada, de acuerdo con la edición, país de origen, autor, fecha y eje de la cartografía conceptual que aborda.

3. Se realizó el análisis de la información con base en los ocho ejes de la cartografía conceptual, y con el apoyo de bibliografía para el abordaje del concepto estudiado.

Se envió el trabajo a revisión del especialista y experto de la temática abordada para la mejora y retroalimentación de aquel.

\section{Criterios para la selección de los docu- mentos}

Se emplearon los siguientes criterios en la búsqueda y selección de los documentos para la realización del presente estudio:
1. Se utilizó Google Académico y Redalyc para hacer la búsqueda de documentos que abordan el tema Sistema de Red Social Docente.

2. Se emplearon las siguientes palabras clave esenciales para la búsqueda: plataforma, sitio, red social, docente, profesor.

3. Se seleccionaron los artículos de revistas indexadas que abordan varias o alguna de las preguntas orientadoras.

4. Se seleccionaron artículos entre el 2002 y el 2017. También se analizaron algunos pocos documentos de fechas anteriores por su relevancia.

\section{Documentos analizados}

En total se analizaron 41 documentos, de los cuales 36 corresponden a artículos de investigación que cumplieron las condiciones de búsqueda y 5 corresponden a libros (ver tabla 2).

Tabla 2.

Documentos claves que abordan el concepto "Sistema de Red Social Docente".

\begin{tabular}{|c|c|c|c|c|}
\hline $\begin{array}{c}\text { Tipo de } \\
\text { documento }\end{array}$ & País & Referencia & $\begin{array}{c}\text { Categoría de } \\
\text { análisis que } \\
\text { contempla }\end{array}$ & Indexación y editorial \\
\hline Artículo & Costa Rica & Abarca, 2013. & $\begin{array}{c}\text { Noción y } \\
\text { diferenciación }\end{array}$ & SciELO Citation Index, Redalyc. \\
\hline Libro & Italia & $\begin{array}{c}\text { Adell y Castañeda, } \\
2010 .\end{array}$ & Categorización & Università degli Studi Roma Tre. \\
\hline Artículo & México & Area, 2008. & Clasificación & Latindex, Dialnet, EBSCO, Redalyc. \\
\hline Libro & Estados Unidos & Baker, 2011. & Vinculación & Teachers College. \\
\hline Artículo & Estados Unidos & $\begin{array}{c}\text { Boyd y Ellison, } \\
2007 .\end{array}$ & Noción & Oxford Index, Scopus. \\
\hline Artículo & España & Buxarrais, 2016. & Diferenciación & Scopus. \\
\hline Artículo & España & Cacheiro, 2011. & Vinculación & $\begin{array}{c}\text { Latindex, Dialnet, EBSCO, Redalyc, } \\
\text { Emerging Sources Citation Index. }\end{array}$ \\
\hline Artículo & España & $\begin{array}{c}\text { Cantón, Cañón y } \\
\text { Grande, 2017. }\end{array}$ & Categorización & $\begin{array}{c}\text { Latindex, Dialnet, EBsco, Redalyc, } \\
\text { Emergin Sources Citation Index. }\end{array}$ \\
\hline Libro & España & $\begin{array}{c}\text { Castañeda y Adell, } \\
2013 .\end{array}$ & Categorización & Marfil. \\
\hline México & Castillejos, Torres y & Categorización y \\
vinculación & $\begin{array}{c}\text { Conacyt, Latindex, EBSCO, Redalyc, } \\
\text { SciELO Citation Index, sis. }\end{array}$ \\
\hline
\end{tabular}




\begin{tabular}{|c|c|c|c|c|}
\hline Libro & México & $\begin{array}{l}\text { Cobo y Pardo, } \\
2007 .\end{array}$ & Vinculación & Flacso. \\
\hline Artículo & Estados Unidos & $\begin{array}{l}\text { Coburn y Russell, } \\
2008\end{array}$ & Diferenciación & $\begin{array}{c}\text { Journal Citation Reports, Scopus, } \\
\text { EBSCO, Social Sciences Citation } \\
\text { Index. }\end{array}$ \\
\hline Artículo & España & $\begin{array}{l}\text { Cornejo y Tapia, } \\
2011 .\end{array}$ & Noción & $\begin{array}{c}\text { Latindex, Redalyc, SISBI, EBSCO y } \\
\text { Dialnet. }\end{array}$ \\
\hline Artículo & Venezuela & $\begin{array}{l}\text { Diez de Tancredi, } \\
2016 .\end{array}$ & $\begin{array}{l}\text { Clasificación y } \\
\text { ejemplificación }\end{array}$ & $\begin{array}{l}\text { Latindex, Dialnet SciELO Citation } \\
\text { Index, Redalyc. }\end{array}$ \\
\hline Artículo & Argentina & $\begin{array}{l}\text { Fainholc, Nervi, } \\
\text { Romero y Halal, } \\
2013 . \\
\end{array}$ & Categorización & Latindex. \\
\hline Artículo & Costa Rica & Hütt, 2012. & Clasificación & $\begin{array}{l}\text { SciELO Citation Index, Redalyc, } \\
\text { InfoBase Index. }\end{array}$ \\
\hline Artículo & Estados Unidos & $\begin{array}{c}\text { Johnson, Lustick y } \\
\text { Kim, } 2011 .\end{array}$ & Diferenciación & ERIC. \\
\hline Artículo & México & $\begin{array}{l}\text { Juárez, Torres y } \\
\text { Herrera, } 2017 .\end{array}$ & Vinculación & $\begin{array}{l}\text { Conacyt, Latindex, EBSco, Redalyc, } \\
\text { SciELO Citation Index, SIS. }\end{array}$ \\
\hline Artículo & Brasil & $\begin{array}{l}\text { López, Correa y } \\
\text { Rojas, } 2017 .\end{array}$ & Categorización & EBSCO, Dialnet, Redalyc. \\
\hline Artículo & México & $\begin{array}{l}\text { López, Flores, } \\
\text { Espinoza y Rojo, } \\
2017 .\end{array}$ & Noción & $\begin{array}{l}\text { Conacyt, Latindex, EBSCO, Redalyc, } \\
\text { SciELO Citation Index, sIs. }\end{array}$ \\
\hline Artículo & Estados Unidos & Merril, 2002. & Categorización & $\begin{array}{l}\text { Journal Citation Reports/Social } \\
\text { Sciences Edition, Scopus y EBSCO. }\end{array}$ \\
\hline Artículo & Chile & $\begin{array}{l}\text { Mortis, Valdés, } \\
\text { Angulo, García y } \\
\text { Cuevas, } 2013 .\end{array}$ & Categorización & $\begin{array}{c}\text { Emerging Sources Citation Index, } \\
\text { OEI, Latindex, Dialnet, EBSCO, } \\
\text { Redalyc. }\end{array}$ \\
\hline Libro & España & Navarro, 1996. & Categorización & Narcea. \\
\hline Artículo & Brasil & $\begin{array}{l}\text { Navarro, López y } \\
\text { Hernández, } 2017 .\end{array}$ & Categorización & $\begin{array}{l}\text { Scopus, SciELO Citation Index, } \\
\text { Redalyc. }\end{array}$ \\
\hline Artículo & Ecuador & $\begin{array}{l}\text { Ortiz y Robles, } \\
2017 .\end{array}$ & Vinculación & $\begin{array}{c}\text { Emerging Sources Citation, SciELO } \\
\text { Citation Index, Latindex, Dialnet, } \\
\text { EBSCO y Redalyc. }\end{array}$ \\
\hline Artículo & España & $\begin{array}{c}\text { Payà, Duart y } \\
\text { Mengual, } 2016 .\end{array}$ & $\begin{array}{l}\text { Ejemplificación y } \\
\text { metodología. }\end{array}$ & Scopus. \\
\hline Artículo & Venezuela & $\begin{array}{l}\text { Peña, Pérez y } \\
\text { Rondón, } 2010 .\end{array}$ & $\begin{array}{l}\text { Noción, } \\
\text { caracterización y } \\
\text { clasificación } \\
\end{array}$ & Latindex, Dialnet, EBSCO y Redalyc. \\
\hline Artículo & México & $\begin{array}{l}\text { Pérez, Ortiz y } \\
\text { Flores, } 2015 .\end{array}$ & $\begin{array}{c}\text { Noción y } \\
\text { diferenciación }\end{array}$ & $\begin{array}{l}\text { SciELO Citation Index, Redalyc y } \\
\text { Dialnet. }\end{array}$ \\
\hline Artículo & Venezuela & Quintas, 2016. & $\begin{array}{l}\text { Clasificación y } \\
\text { metodología }\end{array}$ & $\begin{array}{l}\text { Latindex, RedALyC, Dialnet, } \\
\text { SciELO Social Science y Scopus. }\end{array}$ \\
\hline Artículo & México & Rangel, 2015. & Categorización & $\begin{array}{l}\text { Latindex, Dialnet, EBSCO, Redalyc y } \\
\text { Emerging Sources Citation Index. }\end{array}$ \\
\hline Artículo & México & $\begin{array}{l}\text { Ruiz y Aguirre, } \\
2013 .\end{array}$ & Categorización & $\begin{array}{c}\text { Conacyt, Latindex, EBSCO, Redalyc, } \\
\text { SciELO Citation Index y sIs. }\end{array}$ \\
\hline Artículos & Perú & $\begin{array}{l}\text { Saboya y Lazo, } \\
2017 .\end{array}$ & Categorización & $\begin{array}{l}\text { SciELO Citation Index, Emerging } \\
\text { Sources Citation Index, Redalyc. }\end{array}$ \\
\hline Artículo & Costa Rica & Salas, 2002. & Categorización & $\begin{array}{c}\text { Redalyc, Scielo, Latindex y } \\
\text { Emerging Sources Citation Index. }\end{array}$ \\
\hline Artículo & España & Sánchez, 2017. & Vinculación & $\begin{array}{c}\text { Emerging Sources Citation Index, } \\
\text { EBSCO y Redalyc. }\end{array}$ \\
\hline
\end{tabular}




\begin{tabular}{|c|c|c|c|c|}
\hline Artículo & Cuba & $\begin{array}{l}\text { Solórzano y García, } \\
2016 .\end{array}$ & $\begin{array}{l}\text { Noción, } \\
\text { categorización, } \\
\text { caracterización y } \\
\text { diferenciación }\end{array}$ & SciELO Citation Index. \\
\hline Artículo & España & Sotomayor, 2010. & Caracterización & $\begin{array}{c}\text { Emerging Sources Citation Index, } \\
\text { Dialnet. }\end{array}$ \\
\hline Artículo & España & $\begin{array}{l}\text { Túñez y Sixto, } \\
2012 .\end{array}$ & Vinculación & $\begin{array}{l}\text { Latindex, Dialnet, EBSCO, } \\
\text { Redalyc, Emerging Sources } \\
\text { Citation Index. }\end{array}$ \\
\hline Artículo & España & $\begin{array}{l}\text { Tuzel y Hobbs, } \\
2017 .\end{array}$ & Diferenciación & $\begin{array}{l}\text { EBSCO, Scopus, Latindex, Dialnet, } \\
\text { Redalyc. }\end{array}$ \\
\hline Artículo & México & Valenzuela, 2013. & Noción & CONACYT, Latindex. \\
\hline Artículo & Inglaterra & Vogel, 2009. & Diferenciación & University College London. \\
\hline Artículos & Argentina & Yedid, 2013. & Vinculación & $\begin{array}{c}\text { SciELO Citation Index, Latindex, } \\
\text { Dialnet, Redalyc. }\end{array}$ \\
\hline
\end{tabular}

Fuente: elaboración propia, 2018.

\section{Resultados y discusión}

A partir de la revisión documental se categorizó la información recabada para lograr la comprensión del concepto Sistema de Red Social Docente, de acuerdo con los ejes de la cartografía conceptual. A continuación, se muestran los resultados obtenidos de esta actividad.

\section{Noción: ¿cuál es la definición de Sistema de Red Social Docente?}

El diccionario Larousse define 'Red' como un conjunto de personas organizadas para llevar a cabo una acción común (García e Induráin, 2012). Mientras que la Teoría General de Sistemas de Ludwig von Bertalanffy, desde un paradigma científico sistémico, considera que una red es un conjunto de diversos elementos (parte del sistema o parte del medio), interrelacionados a través de conexiones, un sistema capaz de ser estructurado bajo un esquema de relaciones que pueden ser unidireccionales o recíprocas, y que se encuentran en un ambiente dado que también interacciona con los elementos del sistema, sus interrelaciones y el sistema en su conjunto (Arnold y Osorio, 1998). Con base en esta concepción, se identifican diferentes tipos de redes: de computadoras, eléctricas, sociales, las que conectan personas, grupos, sistemas, nodos, instituciones, etc. (Solórzano y García, 2016).

Sin embargo, la idea de red social se prefiguró desde el siglo XVIII (Émile Durkheim y Ferdinand Tönnies) como un grupo de individuos que comparten valores y creencias y forman conexiones, vínculos o relaciones sociales formales e instrumentales. Se trata de interacciones denominadas acciones sociales que, en suma, constituyen una realidad que no puede ser explicada en términos individuales (Pérez y Aguilar, 2012). La concepción de nuestros días evoluciona en su complejidad, al vislumbrar la red social como el conjunto dinámico de diversas conexiones entre personas, grupos e instituciones en contexto de infinitas multiplicidades y autopoiesis (Luhmann, 1991). En otras palabras, se contempla la red social como un sistema abierto en construcción permanente, que convoca a individuos que se identifican con las mismas necesidades y problemáticas y que, en un ejercicio complejo, se autoorganizan y reorganizan con fines diversos (Peña, Pérez y Rondón, 2010).

A partir del uso de la World Wide Web y particularmente del surgimiento de la Web 2.0, se agregó un valor tecnológico al significado de las redes sociales (Boyd y Ellison, 2007; Cornejo y Tapia, 2011; Valenzuela, 2013). Desde esta perspectiva, los SRs han sido definidos por diferentes autores como platafor- 
mas virtuales para el establecimiento de conexiones interpersonales; espacios virtuales que permiten a los usuarios crear un perfil de identidad, tanto público como semipúblico, construir listas de contactos de perfiles de otras personas, comunicarse (textual, gráfica, sonora o por videoconferencia) con ellos, compartir información en cualquier formato con la comunidad y visualizar las conexiones realizadas dentro del sistema (Peña, Pérez y Rondón, 2010; Pérez, Ortiz y Flores, 2015,). Estos espacios virtuales adquieren un valor educativo al permitir socializar información, producciones y ayuda académica en comunidad con ubicuidad e inmediatez. Ello ha favorecido a la formación de comunidades en las que los individuos son atraídos por la diversificación de estrategias comunicativas, alternativas a las tradicionales "cara a cara". En las redes sociales educativas, los usuarios comparten sus conocimientos sobre una determinada materia o disciplina, muestran sus trabajos y exponen sus experiencias al conocimiento de los demás para resolver tareas específicas (Abarca, 2013; López, Flores, Espinoza y Rojo, 2017). Tales comunidades pueden estar configuradas por estudiantes (Facebook), estudiantes y docentes (Schoology), docentes (Red Iberoamericana de docentes, Red de Docentes de América Latina y el Caribe) o en sitios donde se permite la interacción de estudiantes, profesores y padres de familia (Tiching).

Particularmente, los SRSD son definidos por Juan José de Haro, creador Eduredes 2009 (Red Social Docente soportada por la tecnología Ning), como sistemas virtuales de intercambio social de experiencias educativas útiles para los docentes, quienes valorarán aprender de sus iguales; con esta misma filosofía, la Red de Docentes de América Latina y el Caribe añade el lema: "nadie enseña a nadie, con humildad para aprender, todos aprendemos de todos". El sitio tiching.com, por su parte, se describe a sí mismo como una red educativa escolar, en la que la persona puede encontrar y compartir todos los recursos educativos necesarios para aprender y enseñar mejor.
Así, los SRSD aparecen en el escenario virtual como espacios que ofrecen recursos y diversos servicios de apoyo para que los profesores puedan utilizarlos con el fin de alcanzar sus metas particulares (Solórzano y García, 2016). Los recursos que se comparten en los SRSD incluyen cursos, documentos en línea, vídeos y noticias, en formato de texto, video e imagen. Algunos de estos son importados a la red desde otros sitios y algunos son creados por los propios docentes participantes de la red.

\section{Categorización: ¿cuál es la categoría su- perior a la que pertenecen los Sistemas de Redes Sociales Docentes?}

Desde finales del siglo XIX y principios del $\mathrm{XX}$, la objetivación del saber, las formas de reproducción y la especialización del conocimiento contribuyeron a constituir las ciencias de la educación (Vicente, 2016). Estas estudian, describen, analizan y explican los múltiples aspectos de los fenómenos educativos en las diversas sociedades y culturas, y el conjunto de conocimientos que aportan teoría y práctica a los procesos formativos humanos.

Las ciencias de la educación se dividen en varias ramas, entre estas se encuentra la tecnología educativa, incluida en el área de la didáctica (Navarro, 1996; Salas, 2002), campo de estudio híbrido o compartido con las áreas de la computación e informática, que abarcan todas aquellas mediaciones tecnológicas elaboradas con el fin de colaborar en los procesos educativos.

Con la aparición de la Web 2.0 han emergido nuevas categorías analíticas disciplinares tecnoeducativas, como es el caso del denominado "Entorno personal de aprendizaje", acepción que surge desde el 2001 en el marco del proyecto Northern Ireland Integrated Managed Learning Environment y se oficializa en el 2004 en el Congreso Anual, dedicado a Entornos Telemáticos Centrados. Ahora, el término se ha generalizado internacionalmente con la sigla PLE (Personal Learning Environment) (Castañeda y Adell, 2013). 
La concepción del PLE comprende los diferentes dispositivos de comunicación (ordenadores portátiles, teléfonos móviles, dispositivos de medios portátiles, etc.), las aplicaciones (lectores de noticias, clientes de mensajería instantánea, navegadores, calendarios, etc.) y los servicios (marcadores sociales, blogs, wikis, podcast, etc.) (Solórzano y García, 2016).

Desde un enfoque más amplio, el PLE es un entorno conformado por el conjunto de herramientas, fuentes de información, conexiones personales y actividades que cada persona utiliza para aprender e informarse (Solórzano y García, 2016); precisamente, este está constituido no solo por entornos tecnológicos, sino además por entornos sociales. Se considera, entonces, que un PLE se compone de tres elementos: las herramientas que uno elige para aprender, los recursos o fuentes de información y la red personal de aprendizaje que cada uno va construyendo. Así, los SRSD se encuentran categorizados dentro de la tecnología educativa y el PLE.

En los SRSD, concebidos como entornos de aprendizaje, en los que se dan interacciones en un diálogo reflexivo en alternancia de roles, a veces se es aprendiz o a veces se enseña a otro (López, Correa y Rojas, 2017; Saboya, y Lazo, 2017). Esta dinámica establece las condiciones ideales de trabajo entre iguales para gestión de la zona de desarrollo próximo (Solórzano y García, 2016; Sotomayor, 2010). Los intercambios que se dan están mediados por artefactos culturales (las plataformas virtuales de red), que funcionan como el eslabón entre los individuos, a nivel individual y social. Cabe señalar que los artefactos culturales y la colaboración intervienen en los procesos de aprendizaje intersubjetivos o externos, los que después inciden en los procesos intrasubjetivos o internos (Ramírez, 2016); procesos que, finalmente, influyen en la construcción de modelos mentales (Moreira, 2017) de quienes son participantes activos de la red.

Así mismo, se produce un entramado en estructura de red distribuida y flexible, en el que sus integrantes aprenden "conectivis- tamente" gracias a la diversidad de opiniones, la nutrición y el mantenimiento de las conexiones, la condición de que el conocimiento pueda residir en dispositivos no humanos y la capacidad humana de reconfigurar sus apreciaciones para aprender más allá del conocimiento que se tiene. Entre otros procesos, los SRSD posibilitan la gestión del aprendizaje autodeterminado, que se refiere a la capacidad de buscar nuevas maneras de solución a un problema, pero no solo se busca otra solución, sino que se reflexiona sobre los valores subyacentes y se rechaza o incluso se reformula el objetivo a resolver, proceso también denominado doble bucle. En este, el aprendiz autodetermina sus objetivos, metodologías, duración y criterios de evaluación de aprendizaje. En los SRSD, las conexiones sociales en red distribuida, dinámica, flexible e impredecible facilitan la configuración de un entorno personal de aprendizaje en red de contactos (personas) $y$ en red de fuentes de información (contenidos) (Castañeda y Adell, 2013).

Además, el aprendizaje en estos ambientes puede seguir los mismos principios prescriptivos de las teorías representativas del diseño instruccional, y que se refieren a: los participantes se dedican a resolver problemas del mundo real; el conocimiento existente se activa como base para nuevos conocimientos; el aprendiz se expone a nuevos conocimientos; el aprendiz aplica nuevos conocimientos, y los nuevos conocimientos se integran en el mundo del aprendiz (Merrill, 2002).

Así, la explicación del hecho educativo que ocurre en los SRSD está asociada a teorías educativas clásicas y emergentes de la revolución digital. Entre las que se encuentran: el aprendizaje sociocultural de Vigostky (1968), el aprendizaje colaborativo mediado por computadora (Navarro, López y Hernández de la Torre, 2017; Peña, Pérez y Rondón, 2010; Sotomayor, 2010), el conectivismo o aprendizaje en red de Siemens, el aprendizaje autodeterminado de Hanse y Kenyo (Heutagogía), el aprendizaje como red (Learning as Network) de CHatti, la red personal (Personal Knowledge Person; PKN, por sus siglas 
en inglés), los modelos educativos de diseño instruccional: Star Legacy de Vanderbilt Learning Technology Center, 4-Mat de McCarthy, los episodios instructivos de Andre, los enfoques múltiples de comprensión de Gardner, la solución colaborativa de problemas de Nelson, los entornos de aprendizaje constructivista de Jonassen, el aprender haciendo de Schank, y el modelo de diseño instruccional de cuatro componentes de Van Merriënboer, en 2008 (Castañeda y Adell, 2013). Pero, para que esto ocurra, son imperantes dos condiciones: que el profesor sea su propio creador de redes de conocimiento (Ortiz y Robles, 2017) y que utilice el doble bucle en su proceso personal de aprendizaje (Castañeda y Adell, 2013, Ortiz y Robles, 2017).

Por lo que el manejo de las SRSD también se puede categorizar como una competencia digital docente (Cantón, Cañon y Grande, 2017; Castillejo, Torres y Lagune, 2016; Fainholc, B., Nervi, H, Romero, R. y Halal, C., 2013; López, Correa y Rojas, 2017; Mortis, Valdés, Angulo, García y Cuevas, 2013; Rangel, 2015; Ruiz y Aguirre, 2013).

Caracterización: ¿cuáles son las características centrales de los Sistemas de Redes Sociales Docentes?

Como se mencionó, al igual que otras SRS, en los SRSD, los miembros interactúan unos con otros independientemente de su ubicación geográfica o temporal, mediante una comunicación propia de los espacios virtuales como perfiles, blogs, mensajería interna (denominada chat o inbox), foros de discusión, grupos o juegos; espacios que permiten construir y mantener, en el mundo virtual, los vínculos que se establecen en ambientes "cara a cara". Los usuarios, motivados por el hecho de exponer o ser receptores de información, se agrupan de manera libre y espontánea o por propio interés o invitación; este proceso genera un crecimiento geométrico de la población que conforma la red (Peña y Rondón, 2010).

Aquella dinámica está caracterizada por tres condiciones (Pérez, Ortiz y Flores, 2015):
1. Independencia en la inscripción y conectividad individual a la red: los usuarios son quienes deciden de manera libre la forma de integrarse a la red.

2. Independencia en la interactividad: los usuarios, a partir de sus intereses y motivaciones particulares, comparten, reciben y transmiten información para comunicarse con los demás; son estos elementos los que contribuyen a su integración de manera no lineal e impredecible.

3. Libertad para integrarse: difundir información o generar contenidos para compartir con los otros usuarios.

Tales condiciones propician tanto el crecimiento social como la vida activa de la red (compartir información y contenido), que son el sine qua non para el funcionamiento y operación de los SRSD.

\section{Diferenciación: ¿cuáles son las particula- ridades distintivas de los Sistemas de Re- des Sociales Docentes? \\ Una característica de los SRSD es que son} espacios entre iguales, configurados por profesionales de la educación y, por ende, interesados en temas formativos. Eventualmente, los aprendizajes adquiridos en la red son de utilidad inmediata en el desarrollo profesional de sus integrantes (Peña, Pérez y Rodón, 2010), y esto podría ser el motivo principal de la incorporación de los docentes a un SRSD. La centralidad de estas redes en el aprendizaje para transformar la realidad, con fines menos "sociales", caracteriza a estas como comunidades principalmente educativas (Abarca, 2013; Buxarrais, 2016; Pérez, Ortiz y Flores, 2015), en las que "los usuarios comparten sus conocimientos sobre una determinada materia o disciplina, muestran sus trabajos y ponen su experiencia a disposición de los demás, ayudándoles en tareas específicas a través de atención personalizada" (Abuin, 2009, citado por Pérez, et ál, 2015). Así, los integrantes de la comunidad generan 
una red de significados que se construyen o configuran colectivamente (Pérez, Ortiz y Flores, 2015, Tuzel \& Hobbs, 2017). Además, el proceso de reunión de personas se da de manera espontánea, impredecible y sistemática, lo que propicia la construcción y adición de conocimientos para lograr un objetivo común (Moreno, 2004b). La caracterización de los SRSD como comunidades educativas, en las que se comparte horizontalmente un capital social y cultural, con independencia y flexibilidad (Coburn y Russell, 2008; Johnson, Lustick y Kim, 2011; Knox, Savage y Harvey, 2006; Rollert, 2017; Vogel, 2009,) —un capital construido en sociedad, fuera de las reglas establecidas, horarios y obligaciones, bajo los criterios de selección de la perspectiva que ofrece el profesional de la educación (Peña, Pérez y Rodón, 2010)—, determina la diferencia crucial con otras SRS como Facebook o Twitter, pues los SRSD sí son repositorios de capital cultural docente.

Cabe señalar que el capital cultural puede ser expresado en tres estados (Bourdieu, 1979): incorporado, objetivado e institucionalizado. Las prácticas culturales son interiorizadas inconscientemente en las personas, como esquemas cognitivos, valóricos $\mathrm{y}$ afectivos, por lo que estas adoptan pensamientos y comportamientos acordes con el capital cultural en que se encuentran inmersas. En función del capital cultural incorporado, el individuo consume textos, pinturas, monumentos, etc. (capital objetivado, posesiones físicas) o cursos, capacitaciones, certificaciones, etc. (capital institucionalizado, que se refiere a la adquisición de un valor jurídicamente garantizado, desde el punto de vista de la cultura, como un certificado o título profesional).

Además, en estas sociedades se vislumbra, más que en otras, el modelo social posindustrial que rompe las concepciones de la educación tradicional, originada y vinculada con la producción industrial. La sociedad del conocimiento se caracteriza por la creación de conocimiento disciplinar, mediante la colaboración, la comunicación y la resolución de problemas comunes en ambientes virtuales y, entre otros procesos, por la formación continua docente en contextos sociolaborales más exigentes, marcados por la incertidumbre y la necesidad del aprendizaje autónomo y continuo (Buxarrais, 2016).

\section{Clasificación: ¿cuáles son los tipos de Sis- temas de Redes Sociales Docentes?}

Los SRSD se pueden albergar en dos tipos de SRS (Area, 2008; Hütt, 2012; Payà, Duart y Mengual, 2016; Quintas, 2016; Valenzuela, 2013):

1. SRSD albergados en plataformas con propósitos generales (Facebook, Google+, Twitter, Whatsapp, Yahoo, etc.); las redes docentes forman un grupo dentro de estas megacomunidades. Por ejemplo, los docentes de Tecnología Educativa de las Escuelas Normales Mexicanas tienen un grupo cerrado en Facebook denominado DGESPE-La Tecnología Informática aplicada a los centros escolares, con 1.031 miembros. Otro ejemplo, son los 20 profesores del Colegio Particular Cayetano Tarruell en Ecuador, quienes formaron un grupo restringido en Yahoo-grupos para enviar circulares y anuncios de sus actividades.

2. SRSD albergados en plataformas construidas con softwares modulables o específicos (Ning, Joomla), que se configuran y diseñan con el propósito específico de formar redes sociales docentes; un ejemplo es Histoedu, una red social vertical que propende hacia la participación de la comunidad educativa en la docencia e investigación de la historia de la educación y el patrimonio educativo.

Asimismo, estos dos tipos se pueden subdividir en:

- Redes autogestadas por el profesorado, ya sea por iniciativa individual o de grupo; en consecuencia, se administran y desarrollan de forma voluntaria: por ejemplo, RedDOLAC, Red de docentes de América Latina y el Caribe, con 34.929 miembros. 
- Redes tuteladas o corporativas de una institución pública o privada: por ejemplo, redesoei, la red de docentes de Iberoamérica de la Organización de Estados Ibero Americanos, con 29.451 participantes y la red española Internetaula del Instituto Nacional de Tecnologías Educativas y de Formación Profesional, con 15.413 docentes inscritos.

\section{Vinculación: ¿cómo se vinculan los Siste- mas de Redes Sociales Docentes con otros enfoques o perspectivas por fuera de la ca- tegorización?}

LOS SRSD se vinculan con conceptos emergentes de los ambientes virtuales como la identidad virtual o digital, el etiquetado de contenido, la curación de contenidos, la net-etiqueta, la colaboración abierta distribuida y la inteligencia colectiva.

Los docentes participan en las plataformas de redes sociales, ya sean abiertas o cerradas, en la interacción comunicativa para publicar abiertamente una información (publicaciones en su perfil) o en una charla privada con otros profesores mediante correos (inbox), chats o foros de discusión; dicha interacción lo define como un "ser digital" con una "identidad digital". Se trata, entonces, de una representación de su persona a partir de la manera en la que participa en las comunidades sociales virtuales a las que pertenece, y a partir de lo que busca y lo que recupera en la red (Peña, Pérez y Rondón, 2010). Aquella identidad es relevante para que los aportes no permanezcan anónimos en las SRS, ya que en toda práctica social, como son los procesos formativos, es importante considerar la cuestión del otro y de su reconocimiento (Ortiz y Robles, 2017).

Dentro de los SRSD, el contenido se puede guardar y después recuperar fácilmente, gracias al etiquetado (Cacheiro, 2010; Cobo y Pardo, 2007). Las etiquetas o tags son un término descriptor del contenido publicado, y pueden ser una palabra o una frase asignada a la publicación por el autor o recolector del recurso. Así, el resultado del etiquetado, la acción de asignar tags al contenido virtual, es un conjunto de etiquetas que forma una "folksonomía", es decir, el conjunto de etiquetas asignadas por la comunidad de usuarios de un sitio web en particular (Yedid, 2013).

Otro proceso vinculado con los SRSD es la selección o filtración de contenido a demanda del usuario, el cual no se puede satisfacer simplemente con el uso de motores de búsqueda; es, por lo tanto, necesario un real proceso denominado "curación de contenido" o "curaduría", definido como "el acto interactivo de investigar, hallar, filtrar, organizar, agrupar, integrar, editar y compartir el mejor y más relevante contenido de un tópico específico en una significativa colección digital online, que podría ser importante para un grupo de gente cuyo sentido del aprendizaje puede ser actualizado en torno a ese tópico" (Posada, 2013, p. 3, citado en Juárez, Torres y Herrera, 2017).

Además, el vínculo axiológico, que es indispensable, se refiere al concepto de net-etiqueta o el seguimiento de un protocolo de comunicación cortés, resguardo de cierto grado de privacidad para el desarrollo sano de una identidad virtual (Castillejos, Torres, y Lagune, 2016; Sánchez, 2017).

Así mismo, la exposición de una problemática en las redes virtuales de soporte profesional adquiere una connotación multidimensional, ya que un cuestionamiento es estimado por una multitud de evaluadores, quienes en corto tiempo ofrecen una gran cantidad de perspectivas de solución; este proceso se ha denominado "colaboración abierta distribuida" o crowdsource (Baker, 2011).

Finalmente, los espacios virtuales de redes sociales, en el marco de una creación colectiva de contenidos, el establecimiento de recursos compartidos y el control de la calidad de forma colaborativa entre los usuarios, son idóneos para el desarrollo y la implementación de un nuevo tipo de alfabetización crítica, colaborativa y creativa: la inteligencia colectiva (Cobo y Pardo, 2007; Túñez y Sixto, 2012). 
Metodología: ¿cómo se aplican los Sistemas de Redes Sociales?

A continuación, se describen los pasos metodológicos para abordar problemas contextualizados con el empleo de los SRSD, de acuerdo con una metodología procedente de los procesos de aprendizaje que estos posibilitan y los conceptos emergentes de la Web
2.0, vinculados a las redes. Esto permite identificar el proceso de construcción de una estrategia de búsqueda en pro de la resolución de un problema de interés docente; se trata de los pasos que los autores han identificado para ofrecer una orientación al docente en sus procesos de formación, actualización y capacitación continua (ver tabla 3).

Tabla 3.

Metodología para el uso de los SRSD

\begin{tabular}{|c|c|}
\hline $\begin{array}{c}\text { Elementos } \\
\text { metodológicos }\end{array}$ & Descripción \\
\hline $\begin{array}{l}\text { Establecimiento de una } \\
\text { identidad virtual. }\end{array}$ & $\begin{array}{l}\text { - Inscripción a un SRSD. } \\
\text { - Creación del perfil. } \\
\text { - Publicación de contenido de interés, ya sea propio o ajeno. }\end{array}$ \\
\hline $\begin{array}{l}\text { Establecimiento } \\
\text { del proceso de } \\
\text { autodeterminación. }\end{array}$ & $\begin{array}{l}\text { - Distinción de una necesidad, dificultad o vacío de conocimiento. } \\
\text { - Determinación de una meta o propósito que se pretende alcanzar. } \\
\text { - Identificación de las asunciones relacionadas con el problema. }\end{array}$ \\
\hline $\begin{array}{l}\text { Recuperación de los } \\
\text { saberes previos. }\end{array}$ & $\begin{array}{l}\text { - Búsqueda en el acervo personal de contenidos relacionados con el problema } \\
\text { a resolver. } \\
\text { - Publicación en el SRSD de contenidos, ya sea de producción propia o ajena, } \\
\text { lo cual fortalece la identidad virtual asumida. }\end{array}$ \\
\hline $\begin{array}{l}\text { Análisis y gestión del } \\
\text { conocimiento. }\end{array}$ & $\begin{array}{l}\text { - Búsqueda de los contactos y contenidos relacionados con el problema a } \\
\text { resolver. } \\
\text { - Establecimiento de una primera comunicación con los contactos identificados. } \\
\text { - Anuncio en la red del problema que se desea resolver para convocar la } \\
\text { participación de otros en el proceso de su resolución. }\end{array}$ \\
\hline Trabajo colaborativo. & $\begin{array}{l}\text { - Establecimiento de los interesados en el problema, en un grupo de trabajo, } \\
\text { para la publicación de propuestas de solución. } \\
\text { - Establecimiento de comunicación individual, por inbox o socialmente, en el } \\
\text { grupo o foro, para intercambiar opiniones y reflexiones en torno al problema } \\
\text { a resolver. }\end{array}$ \\
\hline $\begin{array}{c}\text { Contextualización y } \\
\text { curaduría. }\end{array}$ & $\begin{array}{l}\text { Recolección de todas las propuestas de solución del problema para luego } \\
\text { hacer un filtro, de acuerdo con el contexto del problema de interés, es decir, } \\
\text { con la condición particular en donde se utilizarán las propuestas de solución. }\end{array}$ \\
\hline $\begin{array}{l}\text { Realización del doble- } \\
\text { bucle. }\end{array}$ & $\begin{array}{l}\text { - Se lleva a cabo la identificación, análisis, interpretación y aplicación de } \\
\text { las propuestas de solución del problema, de tal manera, que se convierta en } \\
\text { conocimiento. } \\
\text { - Cuestionamiento de las asunciones subyacentes, valores y creencias } \\
\text { relacionadas con el problema. } \\
\text { - Se evalúa si proseguir o iniciar de nuevo con otro problemática o visión de } \\
\text { la problemática. }\end{array}$ \\
\hline $\begin{array}{l}\text { Planteamiento del } \\
\text { procedimiento de } \\
\text { resolución del problema. }\end{array}$ & $\begin{array}{l}\text { - El desarrollo del proceso de solución del problema se realiza y documenta } \\
\text { de manera lógica y detallada. } \\
\text { - Se escriben, evalúan y concluyen los resultados obtenidos en el proceso de } \\
\text { solución del problema, de forma clara y precisa. }\end{array}$ \\
\hline
\end{tabular}


Socialización del conocimiento.
- Se elaboran reportes textuales, gráficos o multimedia de los resultados y las conclusiones obtenidas.

- Estos reportes se publican en el SRSD y se establece el seguimiento de su propagación y retroalimentación espontánea, para refinar la propuesta de solución o identificar nuevos problemas.

Nota: transversalmente se realiza una comunicación social, siguiendo protocolos de cortesía o net-etiqueta; y en los contenidos publicados se respetan las leyes de derechos de autor.

Fuente: elaboración propia, 2018.

Ejemplificación: ¿cuál puede ser un ejemplo significativo del uso de un Sistema de Red Social Docente, a través de la metodología antes propuesta?

A continuación, se muestra un ejemplo para que los docentes apliquen esta estrategia de resolución de problemas o inquietudes en el ambiente virtual que propicia la Red de Docentes de América Latina y el Caribe
(RedDOLAC) (ver tabla 4). Es una red autogestada, construida con el sistema Ning, en el 2009, por el profesor Henry Alberto Chero en Perú, y surge como una iniciativa de integración tecnológica docente de profesores provenientes de América Latina, el Caribe, España, Portugal y otros. En pocos meses llegó a contar con la participación de 2.917 miembros, con 6.509 participantes en

Tabla 4.

Ejemplo del uso socioformativo de la Red de Docentes de América Latina y el Caribe (RedDOLAC).

\begin{tabular}{|c|c|}
\hline $\begin{array}{c}\text { Elementos } \\
\text { metodológicos }\end{array}$ & Descripción \\
\hline $\begin{array}{l}\text { Establecimiento de una } \\
\text { identidad virtual. }\end{array}$ & $\begin{array}{l}\text { - Ingresar a la Red de Docentes de América Latina y el Caribe } \\
\text { (RedDOLAC), a través del espacio "Mi página". } \\
\text { - Verificar el correo de ingreso mediante el enlace enviado al correo } \\
\text { electrónico de inscripción a la red. } \\
\text { - Completar los datos personales solicitados y adjuntar fotos. } \\
\text { - Configurar el estado de privacidad, de acuerdo con las necesidades } \\
\text { personales. } \\
\text { - Establecer las conexiones con otras redes sociales, según la } \\
\text { preferencia del usuario. }\end{array}$ \\
\hline $\begin{array}{l}\text { Identificación de un } \\
\text { problema a resolver e } \\
\text { inicio de un proceso de } \\
\text { autodeterminación }\end{array}$ & $\begin{array}{l}\text { - Identificación del problema y las palabras clave relacionadas con el } \\
\text { problema a resolver. } \\
\text { - Identificación de las asunciones subyacentes, valores y creencias } \\
\text { asociadas al problema. }\end{array}$ \\
\hline $\begin{array}{l}\text { Recuperación de los } \\
\text { saberes previos. }\end{array}$ & $\begin{array}{l}\text { - Revisar el contenido relacionado con el problema de interés en el } \\
\text { acervo personal, para ser publicado en la red, ya sea propio o extraño. }\end{array}$ \\
\hline $\begin{array}{l}\text { Análisis y gestión del } \\
\text { conocimiento. }\end{array}$ & $\begin{array}{l}\text { - Uso de las palabras clave como criterio de búsqueda en los motores } \\
\text { buscadores de la RedDOLAC en los siguientes espacios: videos, fotos, } \\
\text { grupos y blogs. } \\
\text { - Marcar con netiquetas ("Me gusta” y favoritos) los contenidos de } \\
\text { interés o guardarlos en el marcador social Delicious, con una netiqueta } \\
\text { descriptiva o hashtag. } \\
\text { - Agregar como amigo al contacto que publicó el contenido de interés. } \\
\text { - Agregar como amigo a quien marcó como interesante el contenido } \\
\text { publicado en la fase de saberes previos. } \\
\text { - Utilizar protocolos de comunicación de net-etiqueta. }\end{array}$ \\
\hline
\end{tabular}




\begin{tabular}{|l|l|}
\hline Trabajo colaborativo. & $\begin{array}{l}\text { - Apertura de un tema en el foro de discusión, con la solicitud de } \\
\text { netiqueta descriptiva o hashtag a los comentarios que se establezcan. } \\
\text { - Comunicación, por la bandeja de correo interna, con los docentes } \\
\text { que expresen interés (directamente en comentarios o indirectamente } \\
\text { en publicaciones) sobre la resolución del problema. }\end{array}$ \\
\hline $\begin{array}{l}\text { Contextualización y } \\
\text { curaduría. }\end{array}$ & $\begin{array}{l}\text { - Revisión de los contenidos seleccionados resguardados en el perfil. } \\
\text { - Revisión de los comentarios emitidos en la red por medio de su } \\
\text { identificación, usando las netiquetas propuestas. } \\
\text { - Discriminación de los contenidos e información no relacionada y } \\
\text { alejada del problema. } \\
\text { - Selección de los contenidos de utilidad directa para la solución del } \\
\text { problema, bajo el contexto particular del docente que lo desea aplicar. }\end{array}$ \\
\hline $\begin{array}{l}\text { Realización del doble- } \\
\text { bucle. }\end{array}$ & $\begin{array}{l}\text { - Evaluación de los resultados en función de las asunciones } \\
\text { subyacentes, valores y creencias asociadas al problema. } \\
\text { - Determinación de seguimiento hacia una propuesta de solución o de } \\
\text { la formulación de la problemática bajo una visión diferente. }\end{array}$ \\
\hline $\begin{array}{l}\text { Planteamiento del } \\
\text { procedimiento de } \\
\text { resolución del problema. }\end{array}$ & $\begin{array}{l}\text { - Con base en la contextualización, elaboración de una estrategia de } \\
\text { mejora. } \\
\text { - Documentación de la estrategia de mejora, en texto, video o } \\
\text { imágenes. } \\
\text { - Evaluación de la estrategia de mejora. } \\
\text { - Respeto de los derechos de autor de los contenidos producidos en } \\
\text { esta etapa. } \\
\text { - Uso de protocolos de comunicación de net-etiqueta. }\end{array}$ \\
\hline $\begin{array}{l}\text { Socialización del } \\
\text { conocimiento. } \\
\text { videos, fotos, grupos y blogs. } \\
\text { - Seguimiento a los comentarios recibidos en estos contenidos. } \\
\text { - Valoración de los comentarios para la realimentación de la estrategia } \\
\text { de solución del problema. } \\
\text { - Respeto de los derechos de autor de los contenidos producidos en } \\
\text { esta etapa. } \\
\text { - Uso de protocolos de comunicación de net-etiqueta. }\end{array}$ \\
\hline
\end{tabular}

Nota: transversalmente se realiza una comunicación social, siguiendo protocolos de cortesía o net-etiqueta, y en los contenidos publicados se respetan las leyes de derecho de autor.

Fuente: Elaboración propia, 2018.

el 2011, y actualmente está conformada por 34.929 miembros (15/02/2018); cada día se añaden más profesionales de la educación. Cabe señalar que, en su primer momento, la RedDOLAC fue principalmente latina; después fue más plural con la presencia de países como Portugal, Francia, Canadá y Estados Unidos de América (Diez, 2016).

\section{Conclusiones}

LOS SRSD son plataformas virtuales de la Web 2.0, que permiten albergar redes sociales de estructura distribuida, difusa, autoorgani- zable y flexible (dinámica y reorganizable) en construcción permanente. Estos espacios pueden ser utilizados por los docentes para publicar distintas clases de contenidos educativos (reflexiones, experiencias, necesidades o problemáticas) en cualquier tipo de formato: texto, imagen o video. Se encuentran categorizados dentro de las herramientas que configuran el entorno personal de aprendizaje docente. En las redes docentes, cada usuario va construyendo conocimientos de manera independiente y personalizada en función de sus intereses y necesidades. Los SRSD son una de las herramientas que el 
docente puede elegir para acceder a las fuentes de información y a otros docentes activos que publican o comparten información.

Las interacciones sociales docentes, mediadas por los SRSD, y la disposición de los miembros para aprender y construir con los otros, posibilitan la gestión de aprendizajes colaborativos - a través de computadoras - conectivistas, autodeterminados, de doble-bucle, en red de contenidos, en red de personas, y los que concuerdan con los principios de los modelos constructivistas de diseño instruccional.

Estas herramientas son plataformas virtuales que ofrecen independencia al usuario, de tal manera que este puede interactuar en la red con toda libertad, ya sea como creador de contenido, interlocutor u observador o recuperar los contenidos de su interés y, al mismo tiempo, colaborar en su difusión. El usuario puede, además, entrar a la red cuando lo crea conveniente e interactuar con las personas que considere pertinentes para su formación. Las redes sociales docentes se distinguen de otras SRS por albergar a una comunidad conformada por profesionales de la educación $y$, por ende, interesados en temas formativos, que en última instancia permiten transformar la realidad laboral inmediata. Esta distinción califica a la red docente como una comunidad de aprendizaje, que construye colectivamente un acervo de contenidos formativos docentes de capital cultural, una comunidad que podría convertirse en una sociedad de conocimiento. LOS SRSD se clasifican en plataformas sociales con propósitos generales (Facebook, Google+, Twitter, Whatsapp, Yahoo, etc.) y en las construidas en software específicos (Ning, Joomla) para estos propósitos particulares. Aquellas pueden, a su vez, ser elaboradas por independientes (autogestoras) o por instituciones (tuteladas); se vinculan con otros conceptos emergentes de los ambientes virtuales como son la identidad virtual o digital, los marcadores sociales, el etiquetado de contenido, la curaduría y la inteligencia colectiva.

Un ejemplo de un SRSD es la Red de docentes de América Latina y el Caribe (RedDO-
LAC), red de tipo autogestada, construida con el sistema Ning. Sin embargo, las potencialidades socioformativas de los SRSD se verán diluidas si no se tienen en cuenta algunos factores indispensables para la vida útil de la red. El primero se refiere a la actividad de los usuarios, pues un SRSD de miembros inactivos que no cultiven la red, ya sea por no publicar información o generar contactos, pronto se disolverá. El segundo factor está asociado al comportamiento de los miembros activos, que se deben saber comportar en la comunidad en línea y seguir las normas de cortesía en internet (net-etiqueta), así como compartir con ética los contenidos a través de vías que no infrinjan las leyes de propiedad intelectual.

La intervención docente en los SRSD, a través de un procedimiento coherente con su metodología emergente, contribuye a la construcción de sociedades de conocimiento, es decir, comunidades que trabajan de manera colaborativa en la gestión, construcción y aplicación del conocimiento para la resolución de problemas locales con una visión global, con sentido crítico y compromiso ético, apoyándose en las tecnologías de la información y la comunicación. Por lo que los docentes que estén integrados a una red profesional y aprovechen la dinámica socioformativa que en esta ocurre estarán mejor preparados para afrontar los desafíos que el aula del siglo XXI impone, y percibirán la relevancia de aprender durante toda la vida y de "aprender a aprender" permanentemente. Todas las capacidades que se forman en estas nuevas redes son indispensables para la adquisición de presentes y futuras competencias en un mundo altamente conectado y en constante reconstrucción, debido a aceleradas innovaciones tecnológicas que inmediatamente se ponen en práctica (Redecker, Leis y Leendertse, 2011). Ver a los SRSD más allá de un artefacto tecnológico implica apreciarlos como un ambiente informal de aprendizaje, en el que los flujos de comunicación virtual son un componente indispensable en la formación continua docente. 


\section{Referencias}

Abarca, S. (2013). Las redes sociales como instrumento de mediación pedagógica: Alcances y limitaciones. Revista Electrónica Actualidades Investigativas en Educación, 13(2), 1-18. https://doi.org/10.15517/aie. v13i2.11726

Adell, J., \& Castañeda, L. (2010). "Los Entornos Personales de Aprendizaje (PLEs): una nueva manera de entender el aprendizaje.” En Roig Vila, R. \& Fiorucci, M. (Eds.) Claves para la investigación en innovación y calidad educativas. La integración de las Tecnologías de la Información y la Comunicación y la Interculturalidad en las aulas. Alcoy:Marfil-Roma TRE Universita degli studi. Recuperado de https://digitum.um.es/ jspui/bitstream/10201/17247/1/Adell\&Casta\%C3\%B1eda_2010.pdf

Area, M. (2008). Las redes sociales en internet como espacios para la formación del profesorado. Razón y Palabra, 13(63), 1-11. Recuperado de http://www.redalyc.org/articulo.oa?id=199520798005

Arnold, M., \& Osorio, F. 1998. Introducción a los conceptos básicos de la teoría general de sistemas. Cinta de Moebio: Revista de Epistemología de Ciencias Sociales, 0(3), 40-49. Recuperado de https://cintademoebio.uchile.cl/index.php/CDM/article/ view/26455/27748

Baker, K. (2011). The Networked Teacher: How New Teachers Build Social Networks for Professional Support. NewYork, NY: Teachers College.

Bardin, L. (1996) Análisis de contenido. Madrid: Akal Ediciones.

Bourdieu, P. (1979). La distinction. Paris: Les Éditions de Minuit.

Boyd, D., \& Ellison, N. (2007). Social network sites: Definition, history, and scholarship. Journal of Computer-Mediated Communication, 13(1), 210-230. https://doi. org/10.1111/j.1083-6101.2007.00393.X
Buxarrais, M. (2016). Redes sociales y educación. Teoría de la Educación. Educación y Cultura en la Sociedad de la Información, 17(2), 15-20. http://dx.doi.org/10.14201/ eks20161721520

Cacheiro, M. (2011). Recursos educativos TIC de información, colaboración y aprendizaje. Pixel-Bit. Revista de Medios y Educación, 0(39), 69-81. Recuperado de https://recyt.fecyt.es/index.php/pixel/article/ view/61451

Cantón, I., Cañón, R., \& Grande, M. (2017). La comunicación como subdimensión de la competencia digital en futuros maestros de primaria. Pixel-Bit. Revista de Medios y Educación, 0(50), 33-47. http://dx.doi. org/10.12795/pixelbit.2017.i50.02

Castañeda, L., \& Adell, J. (Eds.). (2013). Entornos Personales de Aprendizaje: Claves para el ecosistema educativo en red. Alcoy: Marfil.

Castillejos, B., Torres, C., \& Lagunes, A. (2016). La seguridad en las competencias digitales de los millennials. Apertura, 8(2), 5469. http://dx.doi.org/10.18381/Ap.v8n2.914

Cobo, C., \& Pardo, H. (2007). Planeta Web 2.0. Inteligencia colectiva o medios fast food. Grup de Recerca d'Interaccions Digitals, Universitat de Vic. México: Flacso. Recuperado de https://www.planetaweb2.net/

Coburn, C., \& Russell, J. (2008). District Policy and Teachers' Social Networks. Educational Evaluation and Policy Analysis, 30(3), 203-235. https://doi. org/10.3102/0162373708321829

Cornejo, M., \& Tapia, M. (2011). Redes sociales y relaciones interpersonales en internet. Fundamentos en Humanidades, 0(24), 219-229. Recuperado de http://fundamentos.unsl.edu.ar/pdf/articulo-24-219.pdf

Diez, D. (2016). Red de Docentes de América Latina y del Caribe -Red DOLAC-. Revista de Investigación, 40(89), 196-197. Recuperado de http://www.scielo.org.ve/pdf/ri/ v40n89/art11.pdf 
Fainholc, B., Nervi, H., Romero, R., \& Halal, C. (2013) La formación del profesorado y el uso pedagógico de las TIC. RED, Revista de Educación a Distancia, 0(38), 1-14. Recuperado de http://www.um.es/ead/red/38

Fernández, E., Rodríguez, C., \& Haya, I. (2017). Análisis de la investigación nacional e internacional sobre redes sociales en contextos educativos. Profesorado. Revista de Currículum y Formación de Profesorado, 21(1), 313-332. Recuperado de https:// recyt.fecyt.es/index.php/profesorado/article/ view/58065/35577

García, A., Froment, F., Bohórquez, R., \& Vieira, L. (2017). Análisis bibliométrico de la interacción profesor-alumno a través de las redes sociales. Pixel-Bit. Revista de Medios y Educación, (51), 53-67. http://dx.doi. org/10.12795/pixelbit.2017.i51.04

García, T., \& Induráin, J. (2012). El pequeño Larousse ilustrado. México: Larousse.

Hütt, H. (2012). Las redes sociales: Una nueva herramienta de difusión. Reflexiones, 91 (2), 121-128. Recuperado de https://revistas.ucr.ac.cr/index.php/reflexiones/article/view/1513/1521

Johnson, W., Lustick, D., \& Kim, M.J (2011). Teacher professional learning as the growth of social capital. Current Issues in Education, 14 (3), 1-13. Recuperado de http://cie.asu.edu/ojs/index.php/cieatasu/article/view/781

Juárez, D., Torres, C., \& Herrera, L. (2017). Las posibilidades educativas de la curación de contenidos: una revisión de literatura. Apertura, 9(2), 116-131. http://dx.doi. org/10.18381/Ap.v9n2.1046

Knox, H., Savage, M. \& Harvey, P. (2006) Social networks and the study of relations: networks as method, metaphor and form. Economy and Society, 35(1), 113-140. http:// dx.doi.org/10.1080/03085140500465899

Krippendorff, K. (1990) Metodología del análisis de contenido. Teoría y práctica. Barcelona: Paidós.
López, L., Correa, L., \& Rojas-Bahamón, M. (2017). Representaciones sociales: formación y uso de tecnologías de información y comunicación. Profesores de educación básica secundaria. Revista Virtual Universidad Católica del Norte, 50, 256276. Recuperado de http://revistavirtual. ucn.edu.co/index.php/RevistaUCN/article/ view/823/1341

López, M., Flores, K., Espinoza, A., \& Rojo, D. (2017). Posibilidades de Facebook en la docencia universitaria desde un caso de estudio. Apertura, 9(2), 132-147. http://dx.doi. org/10.18381/ap.v9n2.1133

Luhmann, N. (1991). Sistemas sociales. México: Editorial Iberoamericana.

Mayring, P. (2000) Qualitative content analysis. Forum qualitative social research, 1(2). http://dx.doi.org/10.17169/fqs-1.2.1089

Mazer, J., Murphy, R., \& Simonds, Ch.(2009). The effects ofteacher self-disclosure viaFacebook on teacher credibility. Learning, Media and Technology, 34(2), 175-183. https://doi. org/10.1080/17439880902923655

Merrill, D, (2002). First principles of instruction. Educational Technology, Research and Development, 50(3), 43-59. https://doi. org/10.1007/BF02505024

Moreira, M. A. (2017). Aprendizaje significativo como un referente para la organización de la enseñanza. Archivos De Ciencias De La Educación, 11(12), e29. https://doi. org/10.24215/23468866e 029

Mortis, S., Valdés, A., Angulo, J., García, R., \& Cuevas, O. (2013). Competencias digitales en docentes de educación secundaria. Municipio de un Estado del Noroeste de México. Perspectiva Educacional, Formación de Profesores, 52(2), 135-153. http://doi.org/10.4151/07189729-Vol.52Iss.2-Art.174

Navarro, L., Cuevas, O., \& Martínez, J. (2017). Meta-análisis sobre educación vía TIC en México y América Latina. REDIE. Revista Electrónica de Investigación Educa- 
tiva, 19(1), 10-19. https://doi.org/10.24320/ redie.2017.19.1.1217

Navarro, M. (1996). Dimensiones tecnológicas de la organización escolar. En Tejedor, F., \& Valcárcel, G. Perspectivas en las nuevas tecnologías en la educación (pp. 215226). Madrid:Narcea.

Navarro, M., López, A., \& Hernández, M. (2017). El trabajo colaborativo en red impulsor del desarrollo profesional del profesorado. Revista Brasileira de Educação, 22(70), 651-667. http://dx.doi.org/10.1590/s141324782017227033

Ortiz, D., \& Robles, D. (2017). Educación como práctica social: la cuestión del otro y su reconocimiento. Sophia, Colección de Filosofía de la Educación, 0(23), 169-192. https://doi.org/10.17163/soph.n23.2017.07

Payà, A., Duart, J., \& Mengual, S. (2016). Histoedu, redes sociales e historia de la educación: el pasado pedagógico desde el presente educativo. Teoría de la Educación. Educación y Cultura en la Sociedad de la Información, 17 (2), 55-72. http://dx.doi. org/10.14201/eks20161725572

Peña, K., Pérez, M., \& Rondón, E. (2010). Redes sociales en Internet: reflexiones sobre sus posibilidades para el aprendizaje cooperativo y colaborativo. Revista de Teoría y Didáctica de las Ciencias Sociales, 0(16), 173-205. Recuperado de http://www.saber. ula.ve/handle/123456789/33627

Pérez, G. (1994). Investigación cualitativa. Retos e interrogantes. Tomo II. Técnicas de análisis de datos. Madrid: La Muralla S. A.

Pérez, G., \& Aguilar, A. (2012). Reflexiones conceptuales en torno a las redes sociales en las redes sociales: Un recorrido de la teoría a las prácticas comunicativas en Facebook, Twitter y Google+. Razón y Palabra, 17(79), 1-37. Recuperado de http://www.razonypalabra.org.mx/N/N79/V79/06_PerezAguilar_V79.pdf

Pérez, M., Ortiz, M., \& Flores, M. (2015). Redes sociales en Educación y propuestas metodológicas para su estudio. Ciencia, Docencia y Tecnología, 26(50), 188-206. Recuperado de http://www.pcient.uner. edu.ar/index.php/cdyt/article/view/53

Pinto, M., \& Gálvez, C. 1996. Análisis documental de contenido: procesamiento de la información. Madrid: Síntesis.

Quintas, N. (2016). La emergencia de las redes sociales académicas: su impacto académico. Opción, 32(10), 517-528. Recuperado de http://produccioncientificaluz. org/index.php/opcion/article/view/21833

Ramírez, M. (2016). La comunicación mediada por computadora: Origen, definición, características e investigaciones educativas. En Avances de investigación en la mejora de la educación en valores y formación de docentes (pp. 26-38). El Fuerte: Universidad Autónoma Indígena de México. Recuperado de https://www. researchgate.net/publication/319967187 La_comunicacion_mediada_por_computadora_Origen_definicion_caracteristicas_e_investigaciones_educativas

Rangel, A. (2015). Competencias docentes digitales: Propuesta de un perfil. Pixel-Bit. Revista de Medios y Educación, 0(46), 235-248. http://dx.doi.org/10.12795/ pixelbit.2015.i46.15

Redecker, A., Leis, M., \& Leendertse, M. (2011). The Future of Learning: Preparing for Change. Seville: Institute for Prospective Technological Studies.

Rollert, K. (2017). Advancing or Inhibiting Educational Opportunity: The Power of New Teachers to Reinforce or Deconstruct Social Reproduction in Urban Schools. The Power of Resistance, 12, 31-57. https://doi.org/10.1108/S1479358X20140000012004

Ruiz, M., \& Aguirre, G. (2013). Quehacer docente, TIC y educación virtual o a distancia. Apertura, 5(2), 108-123. Recuperado de http://www.udgvirtual.udg. $\mathrm{mx} /$ apertura/index.php/apertura/article/ view/412 
Saboya, N., \& Lazo, J. (2017). Redes sociales colaborando con docentes de una universidad privada confesional. Apuntes Universitarios. Revista de Investigación, 7(2), 1-11. http://dx.doi.org/10.17162/au.v7i2.167

Salas, F. (2002). Epistemología, educación y tecnología educativa. Revista Educación, 26(1), 9-18. https://doi.org/10.15517/revedu.v26i1.2873

Sánchez, A. (2017). Educación, privacidad y redes sociales: una reflexión arendtiana. Foro de Educación, 15(23), 7-24. http://dx. doi.org/10.14516/fde.434

SCOPEO. (2009). Formación Web 2.0. España: Universidad de Salamanca. Recuperado de http://scopeo.usal.es/?page id=2355

Solórzano, F., \& García, A. (2016). Fundamentos del aprendizaje en red desde el conectivismo y la teoría de la actividad. Revista Cubana de Educación Superior, 35(3), 98-112. Recuperado de http://www.rces.uh. cu/index.php/RCES/article/view/139

Sotomayor, G. (2010). Las redes sociales como entornos de aprendizaje colaborativo mediado para segundas lenguas (L2). Edutec. Revista Electrónica de Tecnología Educativa, 0(34), a149. http://dx.doi. org/10.21556/edutec.2010.34.423

Tobón, S. (2004). Cartografía conceptual. Islas Baleares. España: Ciber educa.

Tobón, S. (2012). Cartografía conceptual: estrategia para la formación y evaluación de conceptos y teorías. México: CIFE.

Tobón, S. (2013). Formación integral y competencias: pensamiento complejo, currículo, didáctica y evaluación. Bogotá: Ecoe Ediciones.

Tobón, S. (2015). Cartografía conceptual: estrategia para la formación y evaluación de conceptos y teorías. México: CIFE.

Túñez, M., \& Sixto, J. (2012). Las redes sociales como entorno docente: Análisis del uso de Facebook en la docencia universitaria.
Pixel-Bit. Revista de Medios y Educación, 0(41), 77-92. http://dx.doi.org/10.12795/ pixelbit

Tuzel, S., \& Hobbs, R. (2017). El uso de las redes sociales y la cultura popular para una mejor comprensión intercultural. Comunicar, 25(51), 63-72. https://doi.org/10.3916/ C51-2017-06

Valenzuela, R. (2013). Las redes sociales y su aplicación en la educación. Revista Digital Universitaria [en línea], 14(4). Recuperado de http://www.revista.unam.mx/vol.14/ num4/art36/index.html

Veletsianos, G., \& Kimmons, R. (2013). Scholars and faculty members' live experiences in online social networks. The Internet and Higher Education, 16, 43-50. https://doi. org/10.1016/j.iheduc.2012.01.004

Vicente, M. (2016). Ciencias de la Educación: nuevas definiciones profesionales desde la historia reciente. Trabajo y Sociedad, 0(27), 155-176.Recuperado de http://www. scielo.org.ar/scielo.php?script $=$ sci arttext\&pid $=$ S1514-68712016000200010

Vogel, M. (2009). Exploring the conditions for academic teachers ${ }^{\text {ee }}$ informal collegial learning about teaching. A social network approach. Educate , 9(2), 18-36. Recuperado de http://www.unse.edu.ar/trabajoysociedad/\#N\%C3\%BAmero\%2027,\%20Invierno $\% 202017$

Yedid, N. (2013). Introducción a las folksonomías: definición, características y diferencias con los modelos tradicionales de indización. Información, cultura y sociedad, 0(29), 13-2. Recuperado de http://revistascientificas.filo.uba.ar/index.php/ICS/article/ view/673 